\title{
Caesarian Scar Pregnancy - A Diagnostic Dilemma
}

\author{
Pratiksha Gupta, ${ }^{1}$ Anju Huria, ${ }^{2}$ Dilpreet Kaur, ${ }^{2}$ Reeti Mehra' \\ 'Department of Gynecology and Obstetrics, Post Graduate Institute of Medical Sciences and research, ESIC, Basaidarapur, \\ New Delhi, ${ }^{2}$ Department of Gynecology and Obstetrics, Government Medical College \& Hospital, Chandigarh, India.
}

\section{ABSTRACT}

Caesarean scar pregnancy is one of the rarest forms of ectopic pregnancy. Transvaginal ultrasound and color flow Doppler provides a high diagnostic accuracy. A delay in diagnosis and treatment can lead to uterine rupture, major hemorrhage, hysterectomy and serious maternal morbidity and mortality. Early diagnosis can offer treatment options of avoiding uterine rupture and hemorrhage, thus preserving the uterus and future fertility. Primary health care provider should know about this rare entity, because if diagnosed timely, and referral to specialized centre is done without delay will definitely save maternal morbidity and mortality. Management plan should be individualized. Termination of pregnancy is the treatment of choice in the first trimester. Expectant treatment has a poor prognosis because of risk of rupture. In this case report we aim to discuss the associated diagnostic dilemma, most appropriate methods of diagnosis and management, with their implications in clinical practice.

Keywords: scar pregnancy; caesarian section; methotrexate.

\section{INTRODUCTION}

Implantation of an ectopic pregnancy within a previous Caesarean scar is a rare condition. Incidence has increased over the years due to the rise in Caesarean section rates worldwide and especially repeat caesarian section. Caesarean scar pregnancy is potentially lifethreatening if not diagnosed and treated early. It may lead to catastrophic complications, such as uncontrolled hemorrhage and uterine rupture as the pregnancy progresses, which may require hysterectomy and results in subsequent loss of fertility. ${ }^{1,2}$ The outcome is dependent on early diagnosis and timely intervention. We are reporting a case report highlighting the diagnostic dilemma associated with it, errors made by us, different methodology of diagnosis is discussed here with, along with literature review.

\section{CASE REPORT}

A 28 years old woman was referred to Government medical college Chandigarh, with diagnosis of incom- plete abortion. On admission her vitals were stable. She was fourth gravida with previous 2 lower segment Caesarian section, both done for Cephalopelvic disproportion. First cesarean was done 5 years back, second was done 2 years back, in some private hospital. There was no intraoperative or post operative complications, puerperal period was uneventful. Third pregnancy was a medical termination of pregnancy, done for spacing, at 6 weeks gestational age, by surgical method, this was followed by curettage. Previous menstrual history was normal. On admission her general physical examination was within normal limits. Obstetrical Examination was done. On per speculum examination cervix and vagina was apparently healthy, slight fresh blood stained discharge was seen. Per vaginal examination revealed cer- 
vix to be in backward direction, uterus was enlarged to 6 weeks size, mobile, non tender, anteverted, bilateral adnexa was normal. Hematological examination was within normal limits. Transvaginal sonography was done; a crumpled intrauterine Gestational sac of 6 weeks size with fetal node without any cardiac activity was seen. She was planned for surgical evacuation. Dilatation and curettage was done. Scanty curetting were obtained. Repeat ultrasound examination showed similar preoperative finding. Now we planned her for evacuation under ultrasound guidance. Tran's abdominal probe was used; plastic tip suction cannula was inserted through cervical internal os. During evacuation ultrasound picture showed - the cannula was seen inside the endometrial canal and the gestational sac was seen anteriorly at the region of previous cesarean scar, (Figure 1). Now final diagnosis of cesarean scar pregnancy was made and the procedure was abandoned. She was now planned for intramuscular methotrexate. Serum beta HCG level was $1400 \mathrm{miu} / \mathrm{ml}$. Liver function test was normal, hemogram was within normal limits. Single intramuscular injection of methotrexate at the rate of $1 \mathrm{mg} / \mathrm{kg}$ body weight was given. She was discharged on the next day. She was followed up with Transvaginal ultrasound and Serum beta HCG level. Serum beta HCG levels normalized within 9 weeks. Ultrasound picture showed complete resorption of gestational sac within 9 weeks.

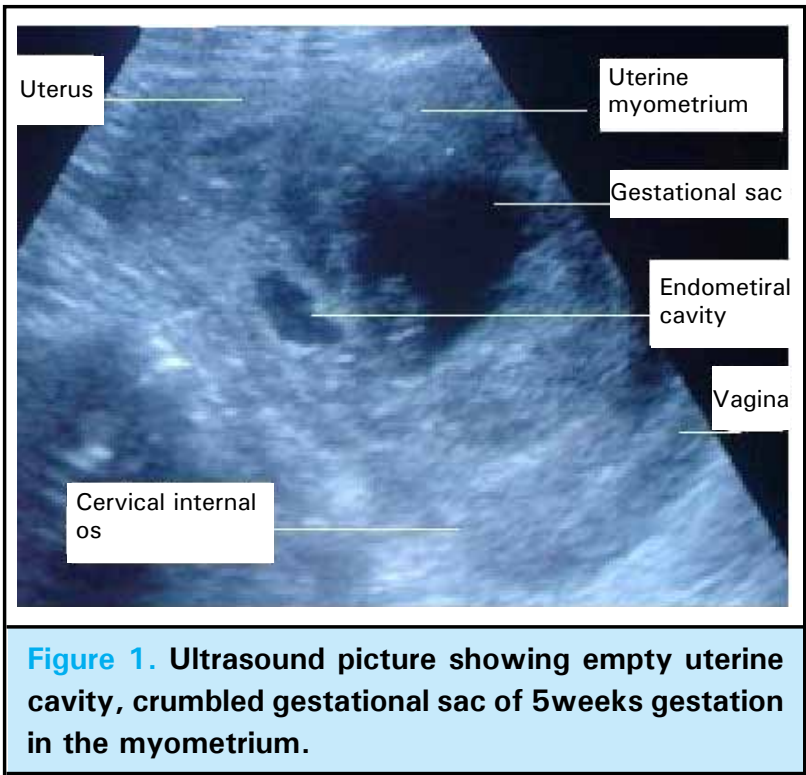

\section{DISCUSSION}

Early recognition of cesarian scar pregnancy is essential because, a delay can lead to increased maternal morbidity and mortality. The first case was reported in the English medical literature in 1978. 1,3 Generally considered among the rarest forms of ectopic pregnancies, its prevalence is estimated between 1 per 1800 and 1 per 2226 pregnancies. ${ }^{1-8}$ However, the incidence is rising with the increased incidence of cesarean deliveries, and the diagnosis is being made earlier because of the increased use of Transvaginal sonography. $3,5,7,9,10 \mathrm{Up}$ to $72 \%$ of cesarean scar pregnancies occur in women who have had 2 or more cesarean deliveries. ${ }^{2,4,7}$ The exact cause and mechanism are not well understood, but it is generally thought that a cesarean scar pregnancy occurs when a blastocyst implants on fibrous scar tissue within a wedge-shaped myometrial defect in the anterior lower uterine segment at the site of a prior cesarean scar. Caesarian scar pregnancies have also been reported after other uterine surgeries such as dilation and curettage, myomectomy, metroplasty, hysteroscopy, and manual removal of the placenta. ${ }^{2,3,9,10}$ The dehiscent myometrial defect may be related to incomplete healing or increased fibrosis along the uterine scar. Fibrosis occurring after multiple cesarean deliveries leads to poor vascularity, which impairs healing. Multiple cesarean deliveries also increases the risk of implantation on the scar, likely due to an increased scar surface area. ${ }^{4,5,7-10}$ Vaginal bleeding and abdominal pain are common presenting symptoms, in first trimester of pregnancy but at least one-third of patients are asymptomatic. Less severe cases often are diagnosed later during the second and third trimesters, it may result in normal live births but with increased maternal morbidity. ${ }^{7,11}$ Early and accurate diagnosis of the condition is crucial, as delay in diagnosis may lead to life-threatening complications such as uterine rupture and massive hemorrhage. As the gestational sac may continue to develop in the caesarian scar, the trends of serum beta-Human Chorionic Gonadotropin (HCG) increment may mimic a viable intrauterine pregnancy. Ultrasound is the first-line diagnostic tool. Early Transvaginal sonography is the reference standard for diagnosis of cesarean scar pregnancies in the first trimester, with $86.4 \%$ sensitivity..$^{2-4,6,8}$ To reduce the risk of a false diagnosis, a combined approach is recommended: a Transvaginal scan to obtain the fine details of the gestation sac and its relation to the scar followed by a meticulous abdominal scan with a full bladder. The latter provides a 'panoramic view' of the uterus and an accurate measurement of the distance between the gestation sac and the bladder. The following sonographic criteria have been put forward for early diagnosis of cesarean scar pregnancies in the first trimester. ${ }^{2-10,12}$ Empty uterus with a clearly visualized endometrium; Empty cervical canal; Gestational sac within the anterior portion of the lower uterine segment at the presumed site of the cesarean scar; thinned or absent myometrium between the gestational sac and bladder. ${ }^{2}$ Other findings include marked peritrophoblastic color Doppler flow around the sac with low-impedance. A negative 'sliding organ sign'- inability to displace the gestational sac from its position at the level of the internal cervical os by gentle pressure applied by 
the Transabdominal probe, for diagnosis has been described. ${ }^{4}$ Magnetic resonance imaging is useful when sonography is inconclusive. Diagnostic Hysteroscopy and laparoscopy can also be used for confirmation. Medical and surgical approaches in treatment have been attempted for preserving fertility, including systemic therapy, local injection, surgical aspiration of the gestational sac, as well as hysteroscopic, laparoscopic, and open removal. 2,5,7,9 Assessment of the anterior uterine wall thickness is essential because a nonsurgical procedure is the most appropriate option when the trophoblast reaches the bladder-uterine space, thereby avoiding an extended operation. ${ }^{7}$ Local, systemic, and combined treatments with methotrexate have been described.6,7,9,13 These approaches are appropriate in women who are hemodynamically stable with an unruptured scar pregnancy of less than 8 weeks gestation and myometrial thickness of less than $2 \mathrm{~mm}$ between the gestational sac and bladder. ${ }^{2,7}$ Systemic methotrexate has a success rate of $71 \%$ to $80 \% .^{2,4}$ Best results are achieved when the $\square$-human chorionic gonadotropin level is less than $5000 \mathrm{mU} / \mathrm{mL}$. 2,6,8 Fibrous tissue within the scar around the gestational sac can delay systemic methotrexate absorption into the sac. Given the short half-life of methotrexate, multiple doses are often required. Local administration of methotrexate, potassium chloride, and hypertonic glucose have all been used successfully. ${ }^{7-9}$ Close monitoring of the patient after intervention is required because hemorrhage may still occur; therefore, concurrent bilateral uterine artery embolization can also be considered. ${ }^{3,7}$ Surgical management with laparoscopy or laparotomy with excision of the pregnancy may be best for women who do not respond to conservative medical treatments or are late to present. ${ }^{14}$ This approach allows for revision of the cesarean scar with new uterine closure that may minimize risk of recurrence. ${ }^{7,9}$ However, surgery carries the risk of postoperative adhesions that may impair future fertility, increased size of surgical wounds, a longer hospital stay and recovery, and possibly an increased risk of future placenta previa/accrete. . $^{2,4,7,8,14}$ The trophoblastic tissue and villi are implanted within the myometrium, so dilation and curettage is unlikely to expel the gestational sac without rupturing the uterine wall or damaging the bladder. Dilation and curettage in patients with cesarean scar pregnancies often leads to massive bleeding requiring emergency laparotomies. ${ }^{2,3,5,7-9}$ Resorption of sac and normalisation of gonadotropin takes 9 weeks to 3 months. . $^{2,4,7}$ To conclude early diagnosis and treatment remains the key for the successful treatment of Cesarian Scar pregnancy. With a high index of suspicion, early diagnosis is possible with ultrasound. Color Doppler ultrasound is important in its early diagnosis and treatment. This diagnosis has to be kept no products of conception are obtained during evacuation, especially when it is being done by primary health care provider, which usually is the case in developing countries like ours. Primary health care provider should know about this rare entity, if diagnosed at appropriate time, and referral to specialized centre will definitely save maternal morbidity and mortality.

\section{REFERENCES}

1. Daniel A. Osborn, Todd R. Williams, Brian M.Craig. Cesarean Scar Pregnancy Sonographic and Magnetic Resonance Imaging Findings, Complications, and Treatment. Journal of Ultrasound in Medicine. 2012;31:1449-56.

2. Ash A, Smith A, Maxwell D. Caesarean scar pregnancy. International Journal of Obstetrics \& Gynaecology. 2007;114:253- 63.

3. Wang CB, Tseng CJ. Primary evcuation therapy for Cesarean scar pregnancy: three new cases and review. Ultrasound Obstet Gynecol. 2006;27:222-26.

4. Jurkovic D, Hillaby K, Woelfer B, Lawrence A, Salim R, Elson CJ. First-trimester diagnosis and management of pregnancies implanted into the lower uterine segment Cesarean section scar. Ultrasound Obstet Gynecol. 2003;21:220-27.

5. Rosen T. Placenta accreta and cesarean scar pregnancy: overlooked costs of the rising cesarean section rate. Clin Perinatol. 2008;35:519-29.

6. McKennaDA,Poder L,Goldman M,Goldstein RB. Role of sonography in the recognition, assessment, and treatment of cesarean scar ectopic pregnancies. J Ultrasound Med 2008; 27:77.
7. Maymon R, Halperin R,Mendlovic S,Schneider D,Herman A. Ectopic pregnancies in a Caesarean scar: review of the medical approach to an iatrogenic complication. Hum Reprod Update. 2004;10:515-23.

8. Seow KM, Huang LW, Lin YH,Lin MY,Tsai YL,Hwang JL. Cesarean scar pregnancy: issues in management. Ultrasound Obstet Gynecol 2004;23:247-53.

9. Fylstra DL. Ectopic pregnancy within a cesarean scar: a review. Obstet Gynecol Surv 2002;57:537-43.

10. Gurel S. Ectopic pregnancy. Ultrasound Clin. 2008;3:331-43.

11. BenNagiJ,Ofili-YeboviD,Marsh M,Jurkovic D. First-trimester cesarean scar pregnancy evolving into placenta previa/ accreta at term. J Ultrasound Med. 2005;24:1569-73.

12. Levine D. Ectopic pregnancy. Radiology 2007;245:385-97.

13. Murray H, Baakdah H, Bardell T, Tulandi T. Diagnosis and treatment of ectopic pregnancy. CMAJ 2005;173:905-12.

14. Wang YL, Su TH, Chen HS. Laparoscopic management of an ectopic pregnancy in a lower segment cesarean section scar: a review and case report. J Minim Invasive Gynecol 2005;12:73-79. 